\title{
SMART CONTROL SOFTWARE FOR INDUSTRIAL PROCESS
}

\author{
EGRI, A[ngela] \& SIRB, V[ali] - C[hivuta]
}

\begin{abstract}
Our goal was to developed, implemented and practical experimented an knowledge-based intelligent software application for control and monitoring of vitally important industrial degassing process from degassing stations. For this purpose we created friendly user interface. This was designed to allow communication between the user and the computer system through a window using images, messages, menus and commands. The data acquired from the process we monitored and driven over with data acquisition boards, Data Acquisition Card and displayed using virtual instruments. The knowledge base is made up by the assembly of all the specialised knowledge introduced by the knowledge expert. Rules base contains basic information necessary to solve the problem (initial facts) and also inferred facts deduced after triggering of the reasoning performed in the inference engine. Keywords: Intelligent control, monitoring, interface, knowledge base, degassing station.
\end{abstract}

\section{INTRODUCTION}

The high complexity of the degassing stations and the necessity to monitor the parameters referring to the : cooling water pressure with water gauge, depression gas extraction, the discharge gas pressure, concentration of methane, presence of water in gas filters due to the condensation process, current absorbed from mains electric vacuum pump ,methane gas pump temperature , the maximum hot water, minimum of hot water ,minimum of hot water ,the presence of methane gas pumps hall, air temperature, detection of methane gas ignition, the maximum water separator, minimum level of water in the separator, impose intelligent monitoring well mathematical based solutions and concretized by the conceiving of the knowledge-based intelligent systems (Egri A., 2002) for control and monitoring for a real industrial degassing stations.

The intelligent system developed and implemented requires the creation of the following components (fig.1): knowledge base, data acquisition module, interface, inference engine, and user interface.

\section{USER INTERFACE OF THE INTELLIGENT SYSTEM}

The user interface is designed to allow communication between the user and the computer system through a window using images, messages, menus and commands. The application software developed and implemented (fig. 2 ) Intelligent Control System main window opens the display of which allows access to menus: Process And Installations (Proc. And Inst.), Acquisition Data Module (D.A.Q. M), Facts Base, Rules Base, Monitoring And Control (C. and M.) with submenus: control panel (Fig. 3), synoptic signals (Fig. 4.) and Layout of the equipment (Fig. 5).

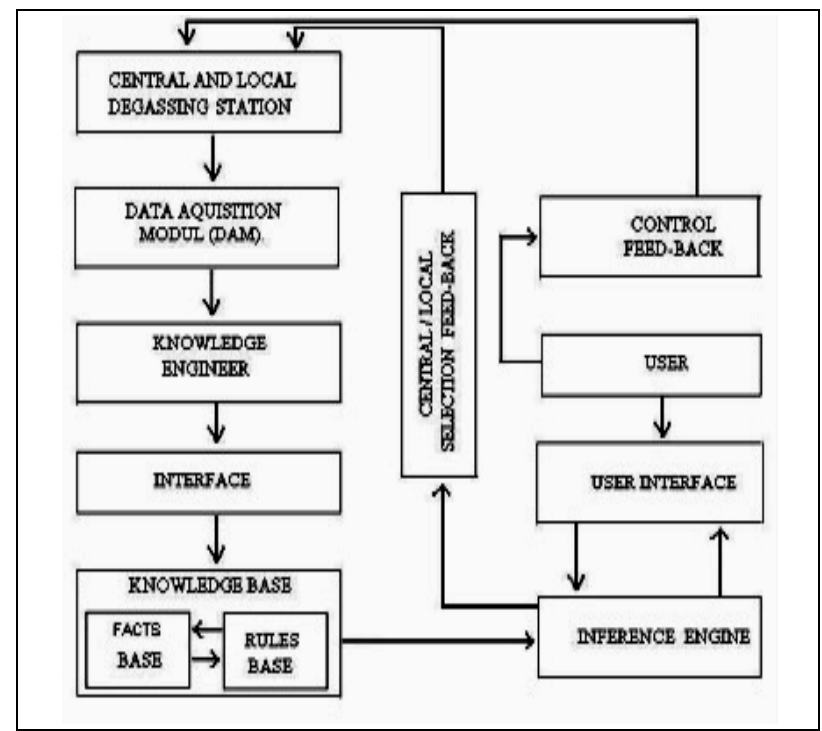

Fig. 1. Architecture of intelligent system

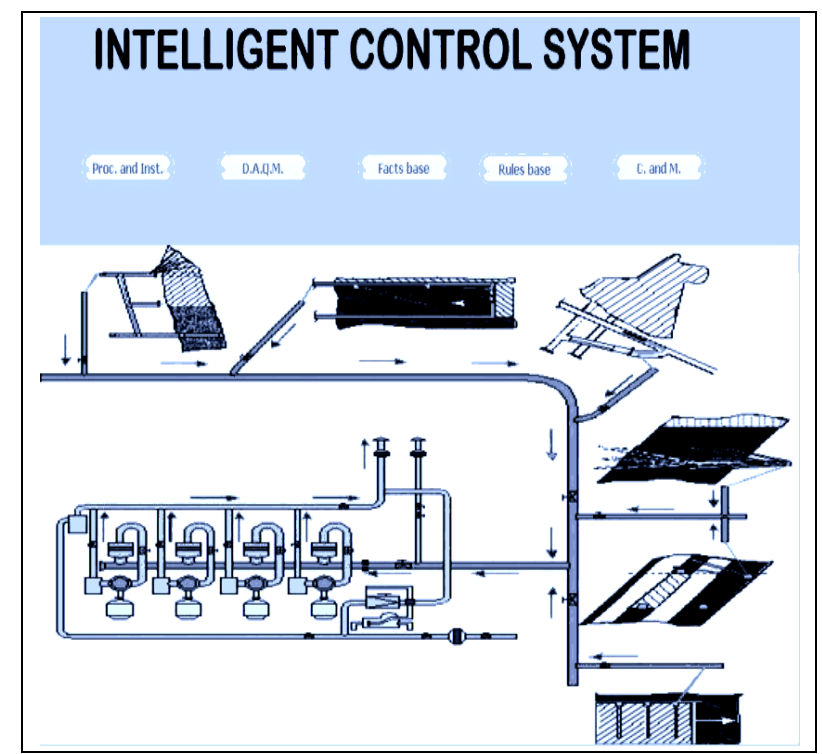

Fig. 2. Intelligent Control System main window

Monitor and adjust parameters were acquired from sensors in the system, they are: Cooling water pressure with water gauge $(\mathrm{Pa})$, Depression Gas Extraction (D), The discharge gas pressure $(\mathrm{P})$, Concentration of methane $\left(\mathrm{CH}_{4}\right.$ analyzer using), Presence of water in gas filters due to the condensation process (FAP), Current absorbed from mains electric vacuum pump (APV), Methane gas pump temperature ( $\mathrm{Tg}$ ), The maximum hot 
water (NMC), Minimum of hot water (Nmc1); Minimum of hot water (Nar), The presence of methane gas pumps hall (MCA), Air temperature (Ta), Detection of methane gas ignition (Ap), The maximum water separator (Nam), Minimum level of water in the separator (NMA).

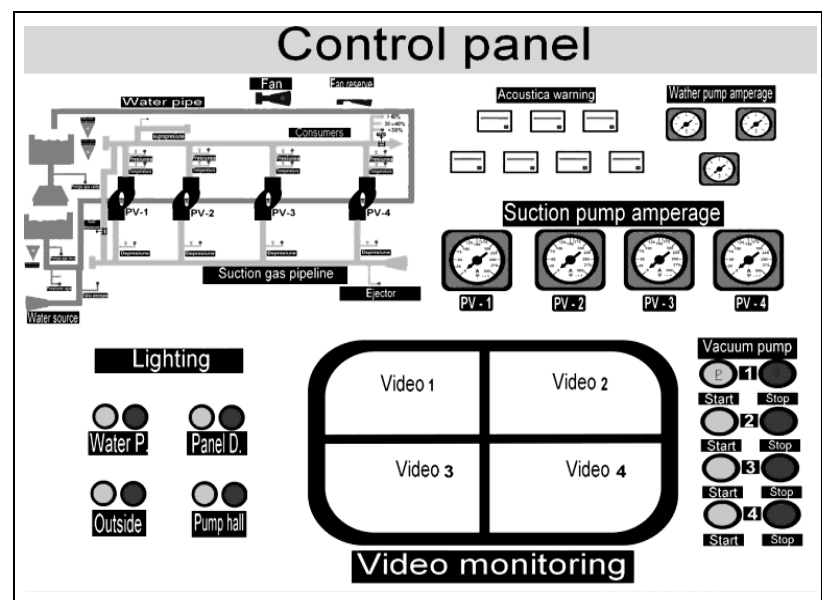

Fig. 3. Control panel

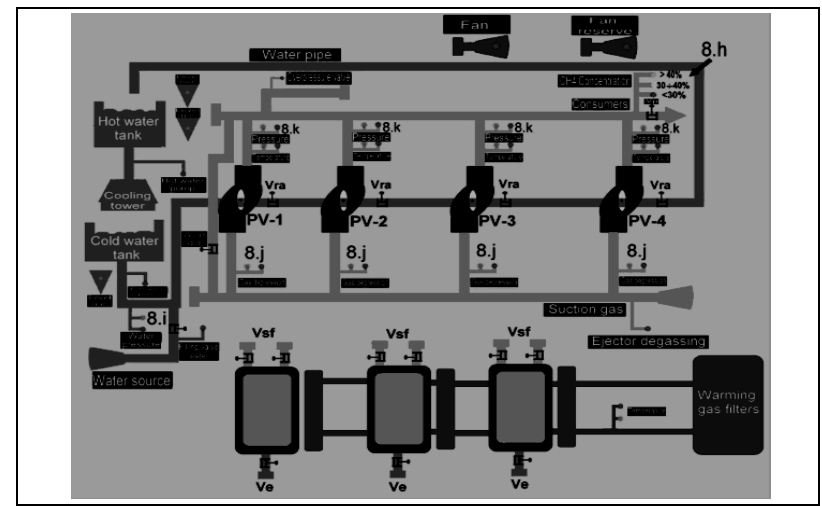

Fig. 4. Synoptic signals

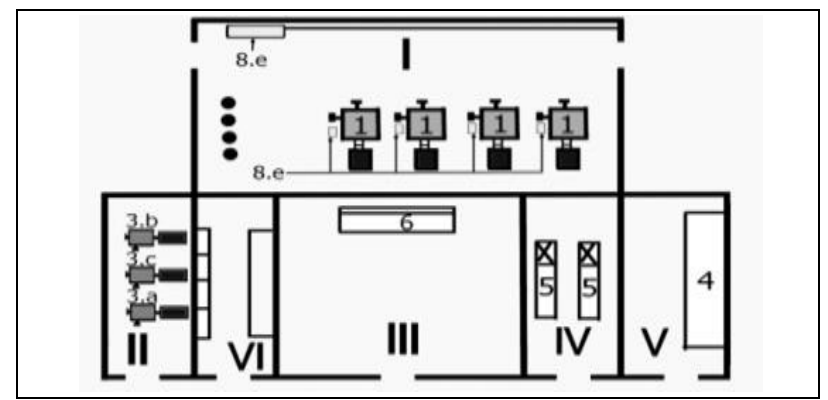

Fig. 5. Layaut of the equipment

\section{DATA ACQUISITION MODULE}

The Data Acquisition Module periodically receives data from connected sensors and sends them to the monitoring system via the knowledge base management system. In order to process the data acquired from the process we monitored and driven over with data acquisition boards, Data Acquisition Card (DAQ) and displayed using virtual instruments were created using LabVIEW graphical programming language. The main window of the interface is presented in fig.6. Signals provided by sensors on the degassing station are analog so it is necessary to convert them into digital signals.

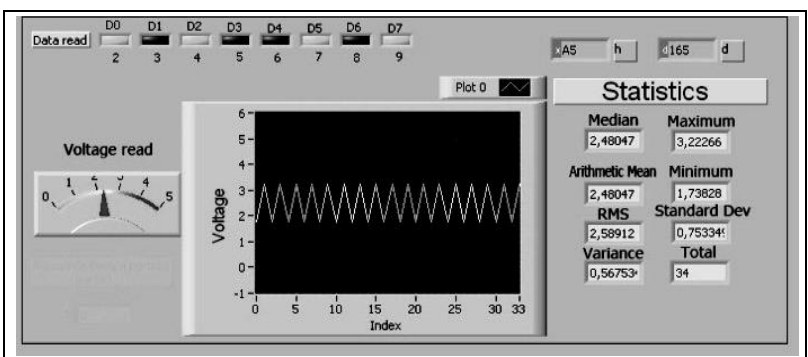

Fig.6. The main window of the interface

The signal is taken using the MyInPort subVI then is multiplied by 5 (reference voltage) and divided by the 256 (number of 8-bit read data (Fig. 7) after that it is read by two indicators one numeric and one of type char. The same signal is converted twice (Fig. 7) from integer to Boolean array values and from matrix to groups of Boolean values (Fig. 8).

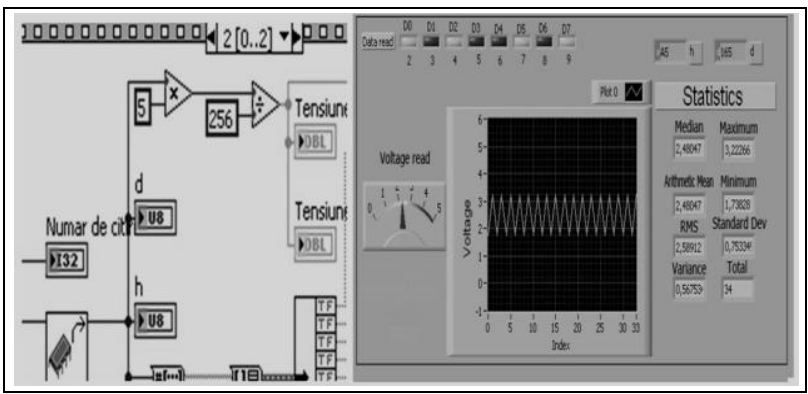

Fig. 7. Signal conversion

The same signal is converted twice (Fig.7) from integer to Boolean array values and from matrix to groups of Boolean values (Fig.8).

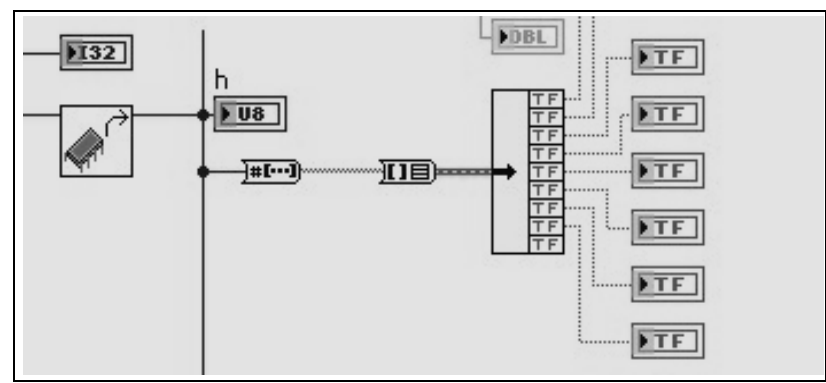

Fig. 8. Boolean matrix creation

These groups will enter an elements separator and will be distributed to one indicator of Boolean values that will serve to indicate on the graphical interface, the time and pins that will used to read data (Fig. 9).

\begin{tabular}{|c|c|c|c|c|c|c|c|c|}
\hline & DO & DI & D2 & D3 & D4 & 05 & D6 & 07 \\
\hline Data read & 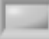 & $=$ & 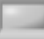 & $=$ & $=$ & 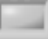 & 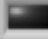 & 2 \\
\hline & 2 & 3 & 4 & 5 & 6 & 7 & 8 & 9 \\
\hline
\end{tabular}

All these processes take place in a while loop (Fig. 10 ), if you press the STOP button the program will exit the loop and send the acquired data to the Statistics subVI (Fig. 11), which will display on the front panel values such as minimum, maximum, arithmetic mean and number of readings. When the program exits the loop the results of the data acquisition will be sent to the FileDialog SubVI and the data will be saved in a user specified file*.xls (Fig.12.) 


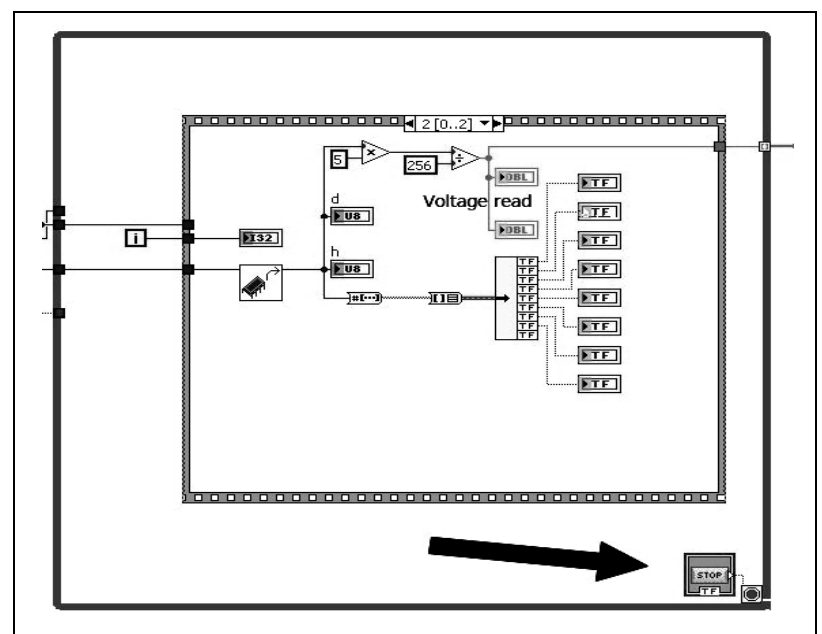

Fig. 10. Process in the loop

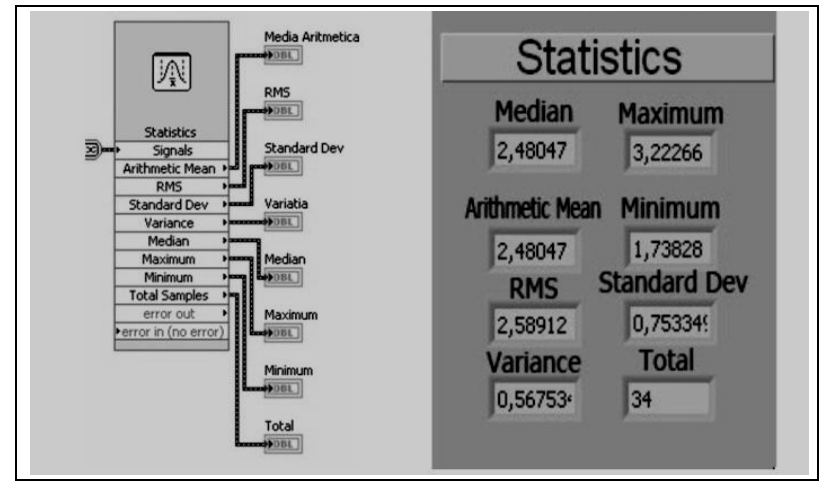

Fig. 11. Statistics

Measurement results were stored in a table (Fig 13), and after with their were generated graphs of variation of parameters (fig14).

\section{THE KNOWLEDGE BASE}

The knowledge base contains two modules: facts base and rules base. The facts are represented using the conventional knowledge representation and the adequate mathematical formalism. The facts (fig 2) is part of the knowledge base (Whitby, B., 2003) and consists of all facts that describe the events taking place both in the central facility and the local degassing station. The Rules base contains basic information necessary to solve the problem (initial facts) and also inferred facts deduced after triggering of the reasoning performed in the inference engine. The inference engine or interpreter is the core of an intelligent system because (Egri A., Sirb V., Patrascoiu N.,TomusA.,2011) it uses the knowledge base to build dynamic reasoning through the selection of trigger table rules based. Rules base contains basic information necessary to solve the problem (initial facts) and also inferred facts deduced after triggering of the reasoning performed in the inference engine.

The inference engine or interpreter is the core of an intelligent system because it uses the knowledge base to build dynamic reasoning through the selection of trigger able rules based on their order of concatenation. For this it is necessary to provide an initial state for the facts base from which we can select trigger able rules, this is the filtering operation. The filtering operation is a selection of rules to be filtered, taking into consideration, initially, a subset of rules, and then selecting the rules applicable
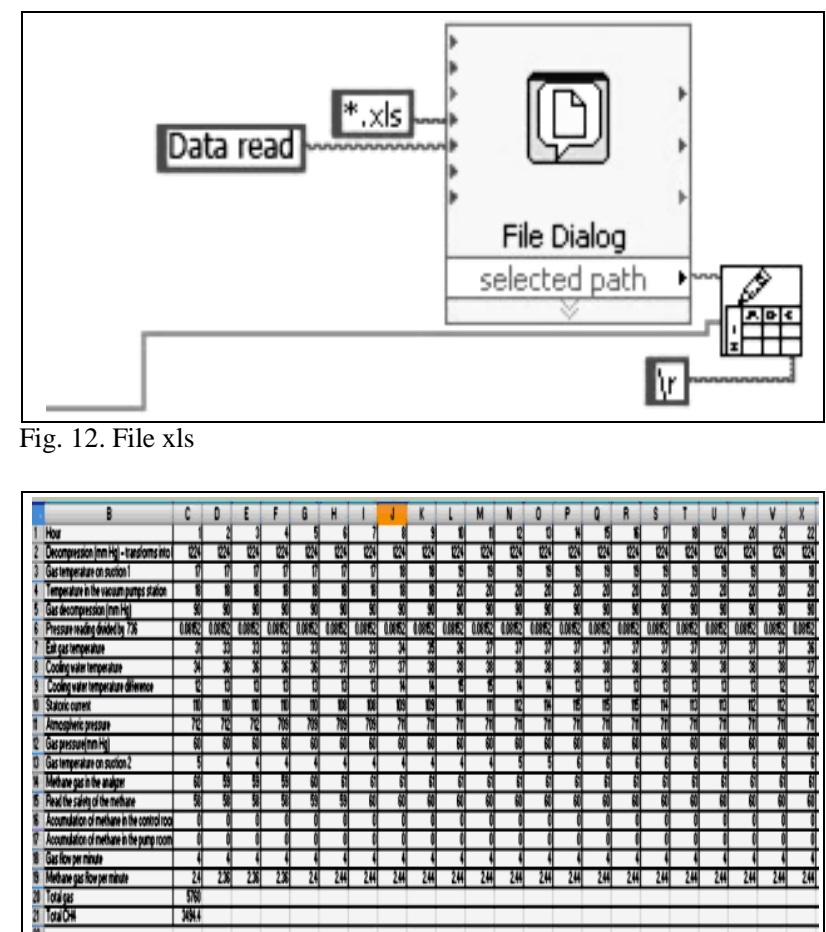

Fig.13 Measurement results

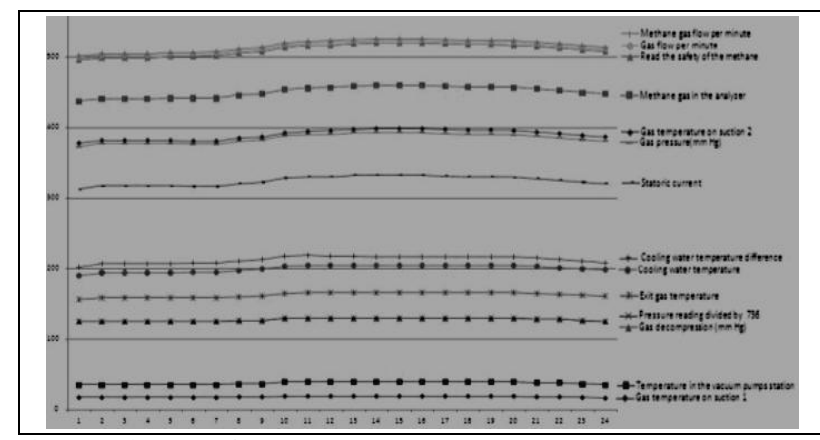

Fig. 14. Diagram of parameters

from this subset. The selection operation performed before filtering is called restriction. Then, the rules that apply are subject to a control strategy through the selection operation. All these operations are performed automatically by the inference engine that will execute the chosen rules. The state of each element is determined by a ,if-then" rule and listed below:

Rule 1: If the water pressure $\mathrm{Pa}(0)$ in the cold water installation doesn't exceed 2 bar then the priming valve $\mathrm{Va}(1)$ of the cold water pump will open and the Par pump will restart.

Rule 2: If the water pressure $\mathrm{Pa}$ (1) of cold water facility exceeds the threshold of 2 bar, the system allows the electric coupling PV of the vacuum pump (1).

Rule 3: If the concentration of methane in facility passes the $30 \%$ threshold $\mathrm{CH}_{4}>30 \%$ (1), the ejector decoupling occurs $\mathrm{Rj}(0)$ and the vacuum pump PV starts (1).

Rule 4: If the concentration of methane in facility has crossed the threshold of $40 \% \mathrm{CH}_{4}>40 \%$ (1) will signal the green synoptic Lv (1) and audible alarms disable Ac (0) of the plant.

Rule 5: If the concentration of methane in facility falls below $40 \% \mathrm{CH}_{4}<40 \%$ (1) the monitoring interface will signal synoptic Lg yellow (1), alarm sound of the plant is turned on Ac (0). 
Rule 6: If the concentration of methane in facility falls below $30 \% \mathrm{CH}_{4}<30 \%$ (1), then the monitoring interface will signal red synoptic LR (1) meaning that the plant will be disengaged automatically, The vacuum pump Pv is decoupled (0) and the ejector $\mathrm{Rj}$ is started (1).

Rule 7: If gas depression $(\mathrm{mmHg})$ is higher than 180 $\mathrm{mmHg} \mathrm{D}>180$ (1) and gas pressure $(\mathrm{mmHg})$ is lower than $10 \mathrm{mmHg} \mathrm{P}<10$ (1), the pressure valve will close $\mathrm{Vp}$ (1) $1 \cdot 10^{-1}$ rot.

Rule 8: If gas depression $(\mathrm{mmHg})$ is higher than 180 $\mathrm{mmHg} \mathrm{D}>180(1)$ and gas pressure $(\mathrm{mmHg})$ is higher 90 $\mathrm{mmHg}$ P> 90 (1), the gas shorting valve opens D (1) $1 \cdot 10^{-1}$ rot

Rule 9: If the water level in the gas filters increases due to condensation, it will open the water exhaust valves Ve (1) after separating the water sections from the extraction circuit by closing partial separation valves USF (1) of the extraction circuits - discharge.

Rule 10: If the current drawn from the network by starting vacuum pump motor absorbs a current greater than the nominal current for 5 seconds Apv> 5 (1), the electrical system will disconnect PV (0) will close the cooling water valve of the pump, it will open the drain valve in the pump Vea (1) and will close the valve on the underground suction pipe Vta (1).

Rule 11: If the gas temperature in the vacuum pump rises above $180 \mathrm{oC} \mathrm{Tg}>1800$ (1), the valve opens cooling

water pump VRA (1) $1 \cdot 4^{-1}$ rot.

Rule 12: If the current drawn from the grid by vacuum pump motor during operation is greater than 160A Apv > 160 (1), then the cooling valve of the vacuum pump closes VRA (1) $1 \cdot 6^{-1}$ rot.

Rule 13: If the pressure in the cold water pump rises above 6 bar Pa.> 6 (1), the shorting valve between the suction and discharge water pumps Vpar opens (1) $1 \cdot 6^{-1}$ rot.

Rule 14: If the water level in the hot water basin reaches the maximum level $\mathrm{Nmc}$ (1), then the maximum level is signaled synoptically on the monitoring interface Lmc (1) and hot water pump will start Pac (1) to push the water to the cooling tower.

Rule 15: If the water level in the hot water basin reaches a set minimum level $\mathrm{Nmc1}$ (1), then the minimum level will be signaled synoptically on the monitoring interface Lmc1 (1) and hot water pump will stop Pac (0).

Rule 16: If the pressure in the cold water pump falls to 2 bar $\mathrm{Pa}=2$ (1), the shorting valve between the suction and discharge water pumps closes Vpar (1) $1 \cdot 6^{-1}$ rot.

Rule 17: If the pressure in the cold water pump falls below 2 bar $\mathrm{Pa}<2$ (1), the vacuum pump will stop PV (0).

Rule 18: If methane collectors detect methane in the pump chamber at a concentration greater than $1.8 \%$ methane $\mathrm{CH}_{4} \mathrm{CMH}>1.8 \%$ (1), the vacuum pump will stop PV (0) and the ventilation fans Vla (1) and dispersion of methane will start.

Rule 19: If the water level in the cold water pool drops below a set lower limit Nar (1), the water supply valve opens Vam (1) to complete.
Rule 20: If the air temperature drops below freezing $\mathrm{Ta}<0^{\circ}$, then will start the "If" filter heating system starts (1).

Rule 21: If methane gas lights Ap (1) due to external factors (electrostatic fields, lightning), it will decouple the degassing station PV (0).

The state of each element is determined by 21 a ,if-then” rule for example:

Rule 22: If the water pressure $\mathrm{Pa}$ (1) of cold water facility exceeds the threshold of 2 bar, the system allows the electric coupling PV of the vacuum pump (1). All rules have been implemented using $\mathrm{C}++$ and were loaded into the controller through the controller interface. Next we present how to implement the R rule (fig. 9).

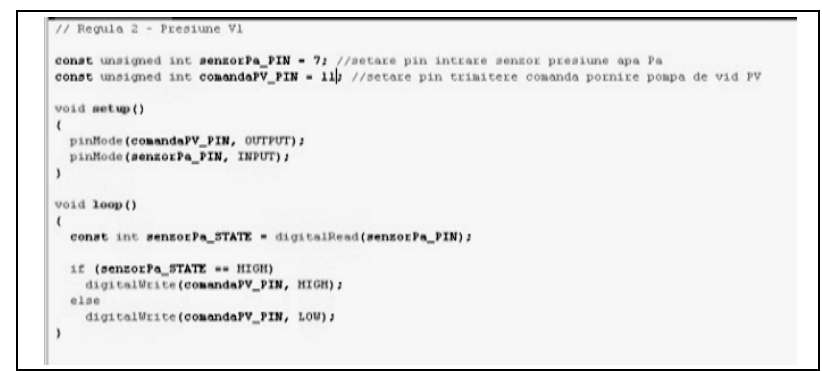

Fig. 9. R2 rule implementation

\section{CONCLUSION}

This smart control software application resolve with high fidelity the control of vitally important industrial degassing process from degassing stations referring to the parameters: cooling water pressure with water gauge, depression gas extraction, the discharge gas pressure ,concentration of methane, presence of water in gas filters due to the condensation process, current absorbed from mains electric vacuum pump ,methane gas pump temperature ,the maximum hot water, minimum of hot water ,minimum of hot water, the presence of methane gas pumps hall ,air temperature, detection of methane gas ignition, the maximum water separator, minimum level of water in the separator.Running quickly and offer very good control and monitoring solutions. The smart software application for control and monitoring of was implemented and experimented .

The software application can be extended to a larger area, more than one degassing stations what we want to do in the future.

\section{REFERENCES}

[1] Alexandru, A.; (2002). Expert systems. Concepts and aplication , Publishing Matrix Rom, ISBN 973-685-226-1, Bucharest, Romania

[2] Benchimol, G. ; Levine, P. \& Pomerol; (1993). Expert systems in the manufactures,Technical Publishing, ISBN 973-31-0471-X, Bucharest, Romania

[3] Egri, A. ; Sirb,V, Patrascoiu,N, Tomus,A.; (2011). Intelligent control and monitoring of drinking water distribution system. Annals of DAAAM for 2011, ISSN 1726-9769, ISBN 978-3901509-83-4, Vienna

[4] Egri, A.; (2002). Artificial intelligence and robotic , Focus Publishing, ISBN 973 -8367 - 48 - 4, Petrosani, Romania

[5] Whitby, B.; (2003). Artificial intelligence , Oneworld Publications, ISBN 1851683224, London, England 